\title{
Reverberation-Ray Matrix Analysis of Acoustic Waves in Multilayered Anisotropic Structures
}

\author{
Yongqiang Guo ${ }^{1}$ and Weiqiu Chen ${ }^{2}$ \\ ${ }^{1}$ Key Laboratory of Mechanics on Disaster and Environment in Western China, Ministry \\ of Education, and School of Civil Engineering and Mechanics, Lanzhou University \\ ${ }^{2}$ Department of Engineering Mechanics, Zhejiang University \\ P.R.China
}

\section{Introduction}

Natural multilayers can be frequently observed, like the layered soils and rocks for example (Kausel \& Roesset, 1981; Kennett, 1983). They are also increasingly used as artificial materials and structures in engineering practices for their high performances (Nayfeh, 1995). For instances, cross-ply and fibrous laminated composites have been applied in naval vessels, aeronautical and astronautical vehicles, and so on for the sake of high strength and light weight (Nayfeh, 1995); piezoelectric thin film systems have been used in various surface acoustic wave (SAW) and bulk acoustic wave (BAW) devices in electronics and information technology in order to accomplish smaller size, lower energy consumption, higher operating frequency and sensitivity, greater bandwidth, and enhanced reception characteristics (Auld, 1990; Adler, 2000). Consequently, as a widespread category of inhomogeneous materials and structures, multilayered structures deserve special concern about their mechanical and acoustical behavior, especially the dynamic behavior since it is what these structures differ most markedly from the homogeneous materials and structures. Investigation of acoustic wave propagation in multilayered structures plays an essential role in understanding their dynamic behavior, which is the main concern in design, optimization, characterization and nondestructive evaluation of multilayered composites (Lowe, 1995; Chimenti, 1997; Rose, 1999) and acoustic wave devices (Auld, 1990; Rose, 1999; Adler, 2000). Nevertheless, the top and bottom surfaces and the interfaces in a multilayered structure cause reflection and/or transmission of elastic waves, giving rise to coupling of various fundamental wave modes in adjacent layers. In multilayered structures consisting of anisotropic media, even the fundamental wave modes themselves are mutually coupled in each layer (Achenbach, 1973). As a result, the analysis of acoustic waves in multilayered structures always remains an extraordinary complex problem, and it is very difficult to obtain a simple and yet numerically well-performed, closed-form analytical solution for a general multilayered structure.

For the above reasons, various matrix formulations have been developed for the analysis of acoustic wave propagation in multilayered media from diverse domains (Ewing et al., 1957; Brekhovskikh, 1980; Kennett, 1983; Lowe, 1995; Nayfeh, 1995; Rose, 1999), since the beginning of this research subject in the midst of last century. Most of these matrix methods 
were presented initially for multilayered structures consisting of isotropic (transversely isotropic) materials, and then extended to those structures made of anisotropic elastic and piezoelectric layers. These matrix methods fall into two groups. One group is those numerical methods based on discrete models, such as the boundary element method (BEM) (Makkonen, 2005), the finite difference method (FDM) (Igel et al., 1995; Makkonen, 2005), the finite element method (FEM) (Datta et al., 1988; Makkonen, 2005) and the hybrid method of BEM and FEM (BEM/FEM) (Makkonen, 2005). This group of methods is powerful for modeling acoustic waves in multilayered structures with various geometries and boundaries. However, they have the disadvantage that the results are approximate, and particularly certain high frequency components must be thrown off in any discrete model. The accuracy of the computational results and the stability of the numerical algorithms depend greatly on the discretization in the temporal and spatial domains. Calculation efficiency will be dramatically decreased if higher accuracy is pursued. The other group is those analytical methods based on continuous (distributed-parameter) model, among which the transfer matrix method (TMM) (Lowe, 1995), also referred to as the propagator matrix method (PMM) (Alshits \& Maugin, 2008), is the typical one. TMM (Thomson, 1950; Haskell, 1953; Lowe, 1995; Nayfeh, 1995; Adler, 1990, 2000) leads to a system equation with dimension keeping small and unchanged as the number of layers increases, since in the formulation the basic unknowns of the intermediate layers are eliminated by matrix products. Thus, TMM has the advantage of high accuracy and high efficiency in most cases, but it suffers from numerical instability in the case of high frequency-thickness products (Nayfeh, 1995; Adler, 1990, 2000; Lowe, 1995; Tan, 2007). Aiming at circumventing this kind of numerical difficulty, different variant forms of TMM as well as analytical matrix methods have been proposed, including the stiffness matrix method (Kausel \& Roesset, 1981; Shen et al., 1998; Wang \& Rokhlin, 2001, 2002a; Rokhlin \& Wang, 2002a; Tan, 2005), the spectral element method (Rizzi \& Doyle, 1992; Chakraborty \& Gopalakrishnan, 2006), the surface impedance matrix method (Honein et al., 1991; Degettekin et al., 1996; Zhang et al., 2001; Hosten \& Castaings, 2003; Collet, 2004), the hybrid compliance/stiffness matrix method (Rokhlin \& Wang, 2002b; Wang \& Rokhlin, 2004a; Tan, 2006), the recursive asymptotic stiffness matrix method (Wang \& Rokhlin, 2002b, 2004b, 2004c), the scattering matrix method (Pastureaud et al., 2002) and the compound matrix method (Fedosov et al., 1996), for instances. Tan (Tan, 2007) compared some of these methods in mathematical algorithm, computational efficiency and numerical stability. However, most of these alternative formulations lack uniformity in a certain degree, and are computationally complicated and inefficient, especially for the high frequency analysis.

Lately, Pao and his coworkers (Pao et al, 2000; Su et al., 2002; Tian et al., 2006) developed the method of reverberation-ray matrix (MRRM) for evaluating the transient wave propagation in layered isotropic and transversely isotropic media. It is shown that MRRM has many advantages and its comparison to the TMM in various aspects was discussed by Pao et al. (Pao et al., 2007). However, the original formulation of MRRM is based on the wave potential functions, which confines this numerically stable and uniform matrix method from extending to layered anisotropic structures. In fact, it is impossible to use wave potential functions for an arbitrarily anisotropic medium, in which the fundamental wave modes are mutually coupled (Achenbach, 1973). Thus, Guo and Chen (Guo \& Chen, 2008a, 2008b; Guo, 2008; Guo et al., 2009) presented a new formulation of MRRM based on state-space formalism and plane wave expansion for the analysis of free waves in anisotropic elastic and piezoelectric layered media. 
The objective of this chapter is to present the general and unified formulation of the method of reverberation-ray matrix (MRRM) for the analysis of acoustic wave propagation in multilayered structures of arbitrarily anisotropic elastic and piezoelectric media based on the state-space formalism and Fourier transforms. In Section 2, the state equation for each layer made of an arbitrarily anisotropic elastic/piezoelectric material is derived from the three-dimensional linear theory of elasticity/piezoelectricity with the help of Fourier transforms, and the solution to the state equation boils down to an eigenvalue problem from which the propagation constants and characteristic mode coefficients can be obtained numerically for a specified frequency. Then the traveling wave solution to the state equation can be written in explicit form in terms of unknown amplitudes as well as known propagation constants and characteristic mode coefficients. In Section 3, we show how the multilayered anisotropic structure is described in both the global and the local dual coordinates. From the boundary conditions on the upper and lower surfaces with applied external forces and the continuity conditions at the interfaces, the scattering relation, which expresses one group of equations for the unknown wave amplitudes in dual local coordinates, is appropriately constructed such that matrix inversion is avoided. Due to the uniqueness of physical essence, the two solutions expressed in dual local coordinates should be compatible with each other, leading to the phase relation, which represents the other group of equations for the unknown wave amplitudes in dual local coordinates. Care must be taken of to properly establish the phase relation such that all exponentially growing functions are excluded. The number of simultaneous equations from the phase and scattering relations amount exactly to the number of unknown wave amplitudes in dual local coordinates, and hence the wave solution can be determined. To reduce the dimension, we substitute the phase relation into the scattering relation to obtain a system equation, from which the dispersion relation for free wave propagation is obtained by letting the determinant of coefficient matrix vanish, and the steady-state and transient wave propagation due to the external force excitations can be obtained by inverse Fourier transforms. Section 4 gives numerical examples of guided wave propagation in cross-ply elastic composite structures. Dispersion curves for different configurations, various boundary conditions and in particular at the high frequency range are illustrated to show the versatility and numerical stability of the proposed formulation of MRRM. Effects of configurations and boundaries on the dispersion spectra are clearly demonstrated through comparison. Conclusions are drawn in Section 5, with highlights of advantages of the proposed general formulation of MRRM for characterizing the acoustic waves in multilayered anisotropic structures.

\section{State space formalism for anisotropic elastic and piezoelectric layers}

\subsection{Governing equations and state vectors}

Consider a homogeneous, arbitrarily anisotropic elastic medium. From the threedimensional linear elasticity (Synge, 1956; Stroh, 1962; Nayfeh, 1995) we have the constitutive relations

$$
\sigma_{i j}=c_{i j k l} \varepsilon_{k l}
$$

the strain-displacement relations

$$
\varepsilon_{k l}=\left(u_{k, l}+u_{l, k}\right) / 2
$$


and the equations of motion (in absence of body forces)

$$
\sigma_{i j, j}=\rho \ddot{u}_{i}
$$

where the comma in the subscripts and superposed dot imply spatial and time derivatives, $\sigma_{i j}, \varepsilon_{k l}$ and $u_{i}$ are respectively the stress, strain and displacement tensors, $c_{i j k l}$ are the elastic constants, and $\rho$ is the material density. The dynamic governing equations can be simplified by eliminating the strain tensor to

$$
\sigma_{i j}=c_{i j k l}\left(u_{k, l}+u_{l, k}\right) / 2 \quad \sigma_{i j, j}=\rho \ddot{u}_{i}
$$

It is commonly difficult to obtain solutions to Eq. (4) for an anisotropic medium of the most general kind as there are 21 independent elastic constants in total, and the deformations in different directions and of different kinds are coupled. However, for an arbitrarily anisotropic elastic layer, the state space formulation (Tarn, 2002a) can be established by grouping the field variables properly. Assume that the correspondence between the digital indices and coordinates follows a usual rule, i.e. $1 \rightarrow x, 2 \rightarrow y$, and $3 \rightarrow z$. If the $z$ axis is along the thickness direction of the laminate, we divide the stresses into two groups: the first consists of the components on the surface of $z=$ const., and the second consists of the remaining components. The combination of the displacements $\mathbf{v}_{u}=[u, v, w]^{\mathrm{T}}$ and the first group of stresses $\mathbf{v}_{\sigma}=\left[\tau_{z x}, \tau_{z y}, \sigma_{z}\right]^{\mathrm{T}}$ gives the state vector $\mathbf{v}=\left[\left(\mathbf{v}_{u}\right)^{\mathrm{T}},\left(\mathbf{v}_{\sigma}\right)^{\mathrm{T}}\right]^{\mathrm{T}}$.

For piezoelectric materials of the most general kind, in the catalogue of three-dimensional linear theory (Ding \& Chen, 2001), we have the constitutive relations instead of Eq. (1)

$$
\sigma_{i j}=c_{i j k l} \varepsilon_{k l}-e_{k i j} E_{k}, D_{i}=e_{i k l} \varepsilon_{k l}+\beta_{i k} E_{k}
$$

the strain-displacement relations of Eq. (2) are further supplemented by

$$
E_{k}=-\varphi_{, k}
$$

and the equations of motion in Eq.(3) are complemented with (in absence of free charges)

$$
D_{i, i}=0
$$

where $D_{i}, E_{k}$ and $\varphi$ are respectively the electric displacement, field and potential tensors, and $e_{k i j}$ and $\beta_{i k}$ are the piezoelectric and permittivity constants, respectively. In view of Eqs. (2), (6) and (5), the dynamic governing equations become

$$
\left\{\begin{array} { l } 
{ \sigma _ { i j } = c _ { i j k l } ( u _ { k , l } + u _ { l , k } ) / 2 + e _ { k i j } \varphi _ { , k } } \\
{ D _ { i } = e _ { i k l } ( u _ { k , l } + u _ { l , k } ) / 2 - \beta _ { i k } \varphi _ { , k } }
\end{array} \quad \left\{\begin{array}{c}
\sigma_{i j, j}=\rho \ddot{u}_{i} \\
D_{i, i}=0
\end{array}\right.\right.
$$

where the coupling between the mechanical and electrical fields is clearly seen. It is noted that the independent piezoelectric and permittivity constants of arbitrarily piezoelectric media should be 18 and 6 respectively, adding further complexity to the solution procedure. However, for an anisotropic piezoelectric layer of the most general kind, the state space formalism (Tarn, 2002b) can also be established just as for arbitrarily anisotropic elastic layer. This will be illustrated in the following section. For piezoelectric materials, the state 
vector is defined by $\mathbf{v}=\left[\left(\mathbf{v}_{u}\right)^{\mathrm{T}},\left(\mathbf{v}_{\sigma}\right)^{\mathrm{T}}\right]^{\mathrm{T}}$, with $\mathbf{v}_{u}=[u, v, w, \varphi]^{\mathrm{T}}$ being the generalized displacements and $\mathbf{v}_{\sigma}=\left[\tau_{z x}, \tau_{z y}, \sigma_{z}, D_{z}\right]^{\mathrm{T}}$ the first group of generalized stresses.

\subsection{Fourier transforms and state equations}

By virtue of the triple Fourier transform pairs as follows

$$
\begin{gathered}
\hat{f}\left(k_{x} ; k_{y}, z ; \omega\right)=\int_{-\infty}^{+\infty} \int_{-\infty}^{+\infty} \int_{-\infty}^{+\infty} f(x, y, z, t) \mathrm{e}^{-\mathrm{i} k_{x} x} \mathrm{e}^{-\mathrm{i} k_{y} y} \mathrm{e}^{-\mathrm{i} \omega t} \mathrm{~d} x \mathrm{~d} y \mathrm{~d} t \\
f(x, y, z, t)=\left(\frac{1}{2 \pi}\right)^{3} \int_{-\infty}^{+\infty} \int_{-\infty}^{+\infty} \int_{-\infty}^{+\infty} \hat{f}\left(k_{x} ; k_{y}, z ; \omega\right) \mathrm{e}^{\mathrm{i} k_{x} x} \mathrm{e}^{\mathrm{i} k_{y} y} \mathrm{e}^{\mathrm{i} \omega t} \mathrm{~d} k_{x} \mathrm{~d} k_{y} \mathrm{~d} \omega
\end{gathered}
$$

the generalized displacements and stresses as well as dynamic governing equations given in Eqs. (4) and (8) in the time-space domain can be transformed into those in the frequencywavenumber domain, where $\omega$ is the circular frequency; $k_{x}$ and $k_{y}$ are the wavenumbers in the $x$ and $y$ directions, respectively; $\mathrm{i}=\sqrt{-1}$ is the unit imaginary; and the $z$ dependent variable in the frequency-wavenumber domain is indicated with an over caret.

By eliminating the second group of generalized stresses, the transformed Eqs. (4) and (8) in a right-handed coordinate system can be reduced to a system of first-order ordinary differential equations with respect to the state vector, which contains $n_{v} / 2$ generalized displacement components and $n_{v} / 2$ generalized stress components, as follows

$$
\frac{\mathrm{d} \hat{\mathbf{v}}(z)}{\mathrm{d} z}=\mathbf{A} \hat{\mathbf{v}}(z)
$$

which is usually referred to as the state equation. The coefficient matrix $\mathbf{A}$ of order $n_{v} \times n_{v}$, with all elements being functions of the material constants, the circular frequency $\omega$ or the wavenumbers $k_{x}$ and $k_{y}$, can be written in a blocked form

$$
\mathbf{A}=\left[\begin{array}{ll}
\mathbf{A}_{11} & \mathbf{A}_{12} \\
\mathbf{A}_{21} & \mathbf{A}_{22}
\end{array}\right]
$$

where

$$
\begin{gathered}
\mathbf{A}_{11}=-\mathrm{i} \mathbf{G}_{33}^{-1} \mathbf{W}, \mathbf{A}_{12}=\mathbf{G}_{33}^{-1}, \mathbf{A}_{22}=-\mathrm{i} \mathbf{W}^{\mathrm{T}} \mathbf{G}_{33}^{-1} \\
\mathbf{A}_{21}=-\rho \omega^{2} \mathbf{M}+k_{x}^{2} \mathbf{G}_{11}+k_{y}^{2} \mathbf{G}_{22}+k_{x} k_{y}\left(\mathbf{G}_{12}+\mathbf{G}_{21}\right)-\mathbf{W}^{\mathrm{T}} \mathbf{G}_{33}^{-1} \mathbf{W}
\end{gathered}
$$

with $\mathbf{W}=k_{x} \mathbf{G}_{31}+k_{y} \mathbf{G}_{32}$. For a layer of arbitrarily anisotropic elastic material, we have

$$
n_{v}=6, \mathbf{G}_{k l}=\left[\begin{array}{lll}
c_{1 k 1 l} & c_{1 k 2 l} & c_{1 k 3 l} \\
c_{2 k 1 l} & c_{2 k 2 l} & c_{2 k 3 l} \\
c_{3 k 1 l} & c_{3 k 2 l} & c_{3 k 3 l}
\end{array}\right], \mathbf{M}=\mathbf{I}_{3}
$$

while for a layer of arbitrarily anisotropic piezoelectric material, we have 


$$
n_{v}=8, \mathbf{G}_{k l}=\left[\begin{array}{cccc}
c_{1 k 1 l} & c_{1 k 2 l} & c_{1 k 3 l} & e_{l 1 k} \\
c_{2 k 1 l} & c_{2 k 2 l} & c_{2 k 3 l} & e_{l 2 k} \\
c_{3 k 1 l} & c_{3 k 2 l} & c_{3 k 3 l} & e_{l 3 k} \\
e_{k 1 l} & e_{k 2 l} & e_{k 3 l} & -\beta_{k l}
\end{array}\right], \mathbf{M}=\left[\begin{array}{cc}
\mathbf{I}_{3} & 0 \\
0 & 0
\end{array}\right]
$$

where $\mathbf{I}_{3}$ denotes the identity matrix of order 3.

\subsection{Traveling wave solutions to the state equation}

According to the theory of ordinary differential equation (Coddington \& Levinson, 1955), the solution to the state equation (11) can be expressed as

$$
\hat{\mathbf{v}}(z)=\boldsymbol{\Phi} \exp (\boldsymbol{\Lambda} z) \mathbf{w}=\left\{\begin{array}{c}
\hat{\mathbf{v}}_{u}(z) \\
\hat{\mathbf{v}}_{\sigma}(z)
\end{array}\right\}=\left\{\begin{array}{l}
\boldsymbol{\Phi}_{u} \\
\boldsymbol{\Phi}_{\sigma}
\end{array}\right\} \exp (\boldsymbol{\Lambda} z) \mathbf{w}
$$

where $\exp (\cdot)$ denotes the matrix exponential function; $\boldsymbol{\Lambda}$ and $\boldsymbol{\Phi}$ are the $n_{v} \times n_{v}$ diagonal eigenvalue matrix and square eigenvector matrix of the coefficient matrix $\mathbf{A}$, respectively; $\boldsymbol{\Phi}_{u}$ and $\boldsymbol{\Phi}_{\sigma}$ are the $n_{v} / 2 \times n_{v}$ sub-matrices of $\boldsymbol{\Phi}$ corresponding to the generalized displacement and stress vectors, respectively; and $\mathbf{w}$ is the vector of undetermined coefficients with $n_{v}$ components.

When combined with the common factors in the integrand of inverse transform in Eq. (10), the solutions in Eq. (16) are interpreted as the total response corresponding to harmonic plane traveling waves with different wavenumbers $-\lambda_{i}$ in the $z$ direction $\left(i=1,2, \cdots, n_{v}\right)$ and common wavenumbers $-k_{x}$ and $-k_{y}$ in the $x$ and $y$-directions at a common radian frequency $\omega$, where $\lambda_{i}$ is the $i$ th component of the diagonal wavenumber matrix $\Lambda$. The corresponding undermined coefficient $w_{i}$ denotes the wave amplitude, and the components of the corresponding eigenvector $\boldsymbol{\Phi}_{i}$ give the state variable response coefficients of the $i$ th wave. According to the nature of wavenumber $-\lambda_{i}$, the characteristic waves can be divided into two groups, the $n_{a}$ arriving waves with their $z$-axis projection along the negative direction and the $n_{d}$ departing waves with their $z$-axis projection along the positive direction (Ingebrigtsen \& Tonning, 1969). Denote the respective sub eigenvalue matrices as $\boldsymbol{\Lambda}_{-}$(of order $n_{a} \times n_{a}$ ) and $\boldsymbol{\Lambda}_{+}$(of order $n_{d} \times n_{d}$ ), both being diagonal, the respective sub eigenvector matrices as $\boldsymbol{\Phi}_{-}$(of order $n_{v} \times n_{a}$ ) and $\boldsymbol{\Phi}_{+}$( of order $n_{v} \times n_{d}$ ), and the respective amplitude vectors as a (the arriving wave vector) and $\mathbf{d}$ (the departing wave vector). Obviously, we have $\mathbf{w}=\left[\mathbf{a}^{\mathrm{T}}, \mathbf{d}^{\mathrm{T}}\right]^{\mathrm{T}}$. Therefore, the matrices $\boldsymbol{\Lambda}_{-}$and $\boldsymbol{\Phi}_{-}$and the vector a correspond to the eigenvalues $\lambda_{i}$, which satisfy $\operatorname{Re}\left(-\lambda_{i}\right)<0$ or $\operatorname{Re}\left(-\lambda_{i}\right)=0, \operatorname{Im}\left(-\lambda_{i}\right)<0$, while the matrices $\Lambda_{+}$ and $\boldsymbol{\Phi}_{+}$and the vector $\mathbf{d}$ are associated with the remaining eigenvalues. It is easily seen that we always have $n_{a}+n_{d}=n_{v}$ with $n_{v}=6$ for elastic materials and $n_{v}=8$ for piezoelectric materials. Consequently, the solution to the state equation given in Eq. (16) can be rewritten as

$$
\begin{aligned}
\hat{\mathbf{v}}(z) & =\left[\begin{array}{ll}
\boldsymbol{\Phi}_{-} & \boldsymbol{\Phi}_{+}
\end{array}\right]\left[\begin{array}{cc}
\exp \left(\boldsymbol{\Lambda}_{-} z\right) & \mathbf{0} \\
\mathbf{0} & \exp \left(\boldsymbol{\Lambda}_{+} z\right)
\end{array}\right]\left\{\begin{array}{l}
\mathbf{a} \\
\mathbf{d}
\end{array}\right\} \\
& =\left\{\begin{array}{c}
\hat{\mathbf{v}}_{u}(z) \\
\hat{\mathbf{v}}_{\sigma}(z)
\end{array}\right\}=\left[\begin{array}{ll}
\boldsymbol{\Phi}_{u-} & \boldsymbol{\Phi}_{u+} \\
\boldsymbol{\Phi}_{\sigma-} & \boldsymbol{\Phi}_{\sigma+}
\end{array}\right]\left[\begin{array}{cc}
\exp \left(\boldsymbol{\Lambda}_{-} z\right) & \mathbf{0} \\
\mathbf{0} & \exp \left(\boldsymbol{\Lambda}_{+} z\right)
\end{array}\right]\left\{\begin{array}{l}
\mathbf{a} \\
\mathbf{d}
\end{array}\right\}
\end{aligned}
$$


where $\boldsymbol{\Phi}_{u-}$ and $\boldsymbol{\Phi}_{\sigma_{-}}$are $n_{v} / 2 \times n_{a}$ sub eigenvector matrices of $\boldsymbol{\Phi}_{-}$corresponding to the generalized displacement and stress vectors, respectively; $\boldsymbol{\Phi}_{u+}$ and $\boldsymbol{\Phi}_{\sigma+}$ are those $n_{v} / 2 \times n_{d}$ sub eigenvector matrices of $\Phi_{+}$. It is noted from Eq. (17) that the only unknowns in the solutions are the wave amplitudes, which should be determined from the system equation formulated by simultaneously considering the dynamic state of all constituent layers of the structure and their interactions. This will be shown in the following section within the framework of reverberation-ray matrix analysis.

\section{Unified formulation of MRRM}

The schematic of a multilayered anisotropic structure of infinite lateral extent is depicted in Fig. 1, which consists of a perfect stacking of $n$ homogeneous arbitrarily anisotropic elastic or piezoelectric layers. From up to down, the layers are denoted in order by numbers 1 to $n$, and the top surface, interfaces and bottom surface in turn are denoted by numbers 1 to $N+1$, bearing the fact that $n=N$. The top and bottom surfaces are denoted by 1 and $N+1$ respectively, and the upper and lower bounding faces of an arbitrary layer, $j$ for instance, are respectively denoted by $J$ and $K$, with $J=j$ and $K=j+1$ and the layer will be referred to as $J K$ or $K J$.

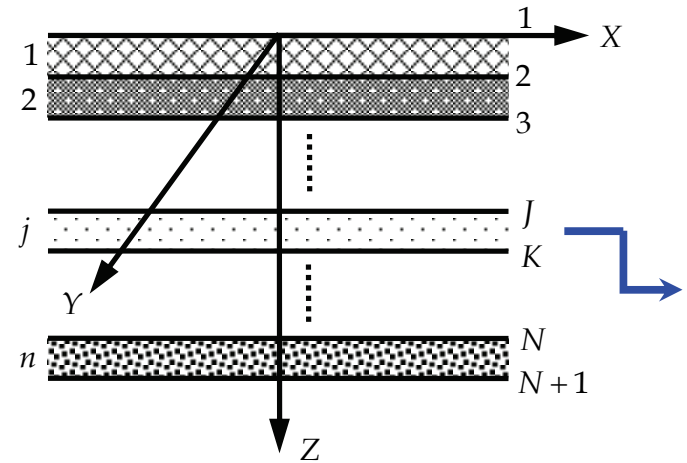

(a) Description of the laminated structure in global coordinates

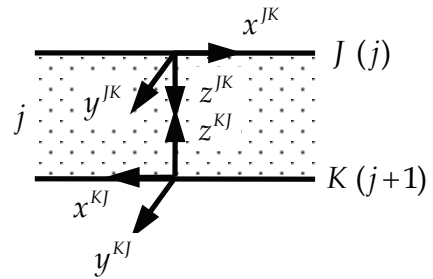

(b) Description of a typical layer $j$ in local dual coordinates

Fig. 1. The schematic of an $n$-layered anisotropic laminate

\subsection{Description of the structural system}

A global coordinate system $(X, Y, Z)$ with its origin located on the top surface and the $Z$ axis along the thickness direction, as shown in Fig. 1a, is established for the system analysis of the whole structure. The physical variables associated with a surface/interface will be described in the global coordinates, and will be affixed with a single superscript, $J$ or $K$ for example, to indicate their affiliation.

As a unique feature of MRRM, a pair of dual local coordinates $\left(x^{J K}, y^{J K}, z^{J K}\right)$ and $\left(x^{K J}, y^{K J}, z^{K J}\right)$ is used to describe each layer $J K$ (or $K J$ ), with $z^{J K}$ originating from $J$ to $K$ and $z^{K J}$ from $K$ to $J, y^{J K}$ and $y^{K J}$ in the same direction as the $Y$-axis, and $x^{J K}$ in the same and $x^{K J}$ in the opposite direction of the $X$-axis, as shown in Fig. 1b. The physical variables 
inside the layers will be described in the local dual coordinates and double superscripts, JK or $K J$ for instance, will be affixed to any physical quantity to denote the corresponding coordinate system and the pertaining layer. As an example, $\hat{\mathbf{v}}^{I K}$ and $\hat{\mathbf{v}}^{K J}$ are the state vectors for layer $J K$ (or $K J$ ) in the coordinates $\left(x^{J K}, y^{J K}, z^{J K}\right)$ and $\left(x^{K J}, y^{K J}, z^{K J}\right)$, respectively. To make the sign convection more clear, physical variables are deemed to be positive as it is along the positive direction of the pertinent coordinate axis.

It is seen from Fig. $1 \mathrm{~b}$ that the dual local coordinates are both right-handed, thus the state equations in Eq. (11) and the traveling wave solutions in Eqs. (16) and (17) all come into existence for an arbitrary layer $J K$ (or $K J)$ in $\left(x^{J K}, y^{J K}, z^{J K}\right)$ and $\left(x^{K J}, y^{K J}, z^{K J}\right)$, which are written as

$$
\begin{gathered}
\frac{\mathrm{d} \hat{\mathbf{v}}^{J K}\left(z^{J K}\right)}{\mathrm{d} z^{J K}}=\mathbf{A}^{J K} \hat{\mathbf{v}}^{J K}\left(z^{J K}\right) \\
\frac{\mathrm{d} \hat{\mathbf{v}}^{K J}\left(z^{K J}\right)}{\mathrm{d} z^{K J}}=\mathbf{A}^{K J} \hat{\mathbf{v}}^{K J}\left(z^{K J}\right) \\
\hat{\mathbf{v}}^{J K}\left(z^{J K}\right)=\boldsymbol{\Phi}^{J K} \exp \left(\boldsymbol{\Lambda}^{J K} z^{J K}\right) \mathbf{w}^{J K}=\left[\begin{array}{ll}
\boldsymbol{\Phi}_{-}^{J K} & \boldsymbol{\Phi}_{+}^{J K}
\end{array}\right]\left[\begin{array}{cc}
\exp \left(\boldsymbol{\Lambda}_{-}^{J K} z^{J K}\right) & \mathbf{0} \\
\mathbf{0} & \exp \left(\boldsymbol{\Lambda}_{+}^{J K} z^{J K}\right)
\end{array}\right]\left\{\begin{array}{l}
\mathbf{a}^{J K} \\
\mathbf{d}^{J K}
\end{array}\right\} \\
\hat{\mathbf{v}}^{K J}\left(z^{K J}\right)=\boldsymbol{\Phi}^{K J} \exp \left(\boldsymbol{\Lambda}^{K J} z^{K J}\right) \mathbf{w}^{K J}=\left[\begin{array}{ll}
\boldsymbol{\Phi}_{-}^{K J} & \boldsymbol{\Phi}_{+}^{K J}
\end{array}\right]\left[\begin{array}{cc}
\exp \left(\boldsymbol{\Lambda}_{-}^{K J} z^{K J}\right) & \mathbf{0} \\
\mathbf{0} & \exp \left(\boldsymbol{\Lambda}_{+}^{K J} z^{K J}\right)
\end{array}\right]\left\{\begin{array}{l}
\mathbf{a}^{K J} \\
\mathbf{d}^{K J}
\end{array}\right\}
\end{gathered}
$$

From Eqs. (20) and (21) we see that there are totally $n_{v} \times N$ arriving wave amplitudes and $n_{v} \times N$ departing wave amplitudes for all layers in dual local coordinates, which should be determined by $2 n_{v} \times N$ relations. It is deduced that the basic unknowns (wave amplitudes) in the MRRM double in number due to the particular description of dynamic state in dual local coordinates, as compared with that in other analytical methods which are usually based on single local coordinates. However, by doing so in the MRRM, the boundary conditions on surfaces and continuous conditions at interfaces take on an extremely simple form since the exponential functions in the solutions no longer appear, as will be seen from Section 3.2. Furthermore, as will be shown in Section 3.3, the arriving and departing wave amplitudes in dual local coordinates are related directly from the point of view of wave propagation through the layer. Thus it shall be possible to deduce a system equation in terms of only the departing wave vectors of all layers. In such a case, the dimension of the system equation will be the same as the one of other analytical methods based on single local coordinates, such as the stiffness matrix method and the spectral element method, as discussed in Section 3.4.

\subsection{Scattering relation from coupling conditions on surfaces and at interfaces 3.2.1 Local scattering relations of top and bottom surfaces}

As depicted in Fig. 2, the response of state variables on the top and bottom surfaces, $\hat{\mathbf{v}}^{12}(0)$ and $\hat{\mathbf{v}}^{(N+1) N}(0)$, corresponding to various waves in the top and bottom layers, respectively, 
should be in accordance with the external state variables $\hat{\mathbf{v}}_{E}^{1}\left(=\left[\left(\hat{\mathbf{v}}_{u E}^{1}\right)^{\mathrm{T}},\left(\hat{\mathbf{v}}_{\sigma E}^{1}\right)^{\mathrm{T}}\right]^{\mathrm{T}}\right)$ and $\hat{\mathbf{v}}_{E}^{(N+1)}$ $\left(=\left[\left(\hat{\mathbf{v}}_{u E}^{(N+1)}\right)^{\mathrm{T}},\left(\hat{\mathbf{v}}_{\sigma E}^{(N+1)}\right)^{\mathrm{T}}\right]^{\mathrm{T}}\right)$, i.e.

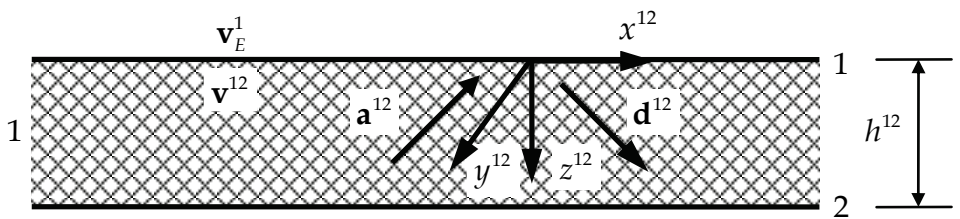

(a) top surface

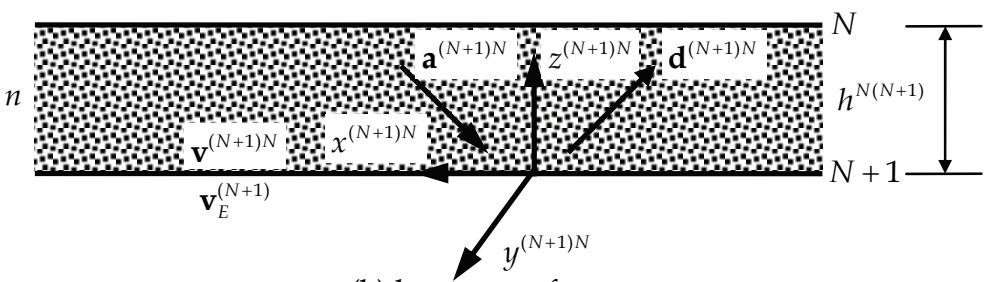

(b) bottom surface

Fig. 2. The top and bottom surfaces of the multilayered anisotropic structure

$$
\hat{\mathbf{v}}^{12}(0)=\mathbf{T}_{E} \hat{\mathbf{v}}_{E}^{1}, \mathbf{T}_{v} \hat{\mathbf{v}}^{(N+1) N}(0)=\mathbf{T}_{E} \hat{\mathbf{v}}_{E}^{(N+1)}
$$

where $\mathbf{T}_{E}=<\mathbf{T}_{u E}, \mathbf{T}_{\sigma E}>$ is a transformation matrix with $\mathbf{T}_{u E}=\mathbf{I}_{n_{v} / 2}$ and $\mathbf{T}_{\sigma E}=-\mathbf{I}_{n_{v} / 2}$; $\mathbf{T}_{v}=<\mathbf{T}_{u}, \mathbf{T}_{\sigma}>$ is also a transformation matrix, with $\mathbf{T}_{u}=<-1,1,-1>$ and $\mathbf{T}_{\sigma}=<1,-1,1>$ for elastic layers and $\mathbf{T}_{u}=<-1,1,-1,-1>$ and $\mathbf{T}_{\sigma}=<1,-1,1,1>$ for piezoelectric layers. Here $<\cdot>$ denotes the (block) diagonal matrix with elements (or sub-matrices) only on the main diagonal and $\mathbf{I}_{n_{v} / 2}$ represents the identity matrix of order $n_{v} / 2$.

By virtue of Eqs. (20) and (21), the solutions to $\hat{\mathbf{v}}^{12}(0)$ and $\hat{\mathbf{v}}^{(N+1) N}(0)$ can be obtained as

$$
\begin{gathered}
\hat{\mathbf{v}}^{12}(0)=\boldsymbol{\Phi}^{12} \mathbf{w}^{12}=\left[\begin{array}{ll}
\boldsymbol{\Phi}_{-}^{12} & \boldsymbol{\Phi}_{+}^{12}
\end{array}\right]\left\{\begin{array}{l}
\mathbf{a}^{12} \\
\mathbf{d}^{12}
\end{array}\right\} \\
\hat{\mathbf{v}}^{(N+1) N}(0)=\boldsymbol{\Phi}^{(N+1) N} \mathbf{W}^{(N+1) N}=\left[\begin{array}{ll}
\boldsymbol{\Phi}_{-}^{(N+1) N} & \boldsymbol{\Phi}_{+}^{(N+1) N}
\end{array}\right]\left\{\begin{array}{l}
\mathbf{a}^{(N+1) N} \\
\mathbf{d}^{(N+1) N}
\end{array}\right\}
\end{gathered}
$$

where the exponential functions disappear since the thickness coordinates on the surfaces are always zero in the corresponding local coordinates. This is the main advantage of introducing the dual local coordinates. It should be noticed that half of the components of vectors $\hat{\mathbf{v}}_{E}^{1}$ and $\hat{\mathbf{v}}_{E}^{(N+1)}$ are known, which are denoted by vectors $\hat{\mathbf{v}}_{K}^{1}$ and $\hat{\mathbf{v}}_{K}^{(N+1)}$, respectively, while the remaining half are unknown, denoted by vectors $\hat{\mathbf{v}}_{U}^{1}$ and $\hat{\mathbf{v}}_{U}^{(N+1)}$, respectively. Substituting Eqs. (23) and (24) into Eq. (22), we can derive

$$
\mathbf{A}^{12} \mathbf{a}^{12}+\mathbf{D}^{12} \mathbf{d}^{12}=\mathbf{T}_{K}^{1} \hat{\mathbf{v}}_{K}^{1}=\mathbf{s}_{0}^{1}, \mathbf{A}^{(N+1) N} \mathbf{a}^{(N+1) N}+\mathbf{D}^{(N+1) N} \mathbf{d}^{(N+1) N}=\mathbf{T}_{K}^{(N+1)} \hat{\mathbf{v}}_{K}^{(N+1)}=\mathbf{s}_{0}^{(N+1)}
$$


where $\mathbf{A}^{12}, \mathbf{D}^{12}, \mathbf{A}^{(N+1) N}, \mathbf{D}^{(N+1) N}, \mathbf{T}_{K}^{1}\left(\mathbf{T}_{K}^{(N+1)}\right)$ are the coefficient matrices with components extracted, in accordance with $\hat{\mathbf{v}}_{K}^{1}$ and $\hat{\mathbf{v}}_{K}^{(N+1)}$, from $\boldsymbol{\Phi}_{-}^{12}, \boldsymbol{\Phi}_{+}^{12}, \boldsymbol{\Phi}_{-}^{(N+1) N}, \boldsymbol{\Phi}_{+}^{(N+1) N}$ and $\mathbf{T}_{E}\left(\mathbf{T}_{E}\right.$ and $\mathbf{T}_{v}$ ) respectively. $\mathbf{s}_{0}^{1}$ and $\mathbf{s}_{0}^{(N+1)}$ are excitation source vectors with $n_{v} / 2$ components of the top and bottom surfaces, respectively. Particularly as far as free waves are concerned, if the top surface is mechanically traction-free (and electrically open-circuit), we have

$$
\begin{aligned}
\hat{\mathbf{v}}_{K}^{1}=\hat{\mathbf{v}}_{\sigma E}^{1}=\left[\hat{\tau}_{X}^{1}, \hat{\tau}_{Y}^{1}, \hat{\sigma}_{Z}^{1}\right]^{\mathrm{T}}=\mathbf{0}\left(\text { or } \hat{\mathbf{v}}_{K}^{1}=\hat{\mathbf{v}}_{\sigma E}^{1}=\left[\hat{\tau}_{X}^{1}, \hat{\tau}_{Y}^{1}, \hat{\sigma}_{Z}^{1}, \hat{D}_{Z}^{1}\right]^{\mathrm{T}}=\mathbf{0}\right) \\
\mathbf{A}^{12}=\boldsymbol{\Phi}_{\sigma-}^{12}, \mathbf{D}^{12}=\boldsymbol{\Phi}_{\sigma+}^{12}
\end{aligned}
$$

and when the top surface is mechanically fixed (and electrically closed-circuit) we have

$$
\begin{aligned}
\hat{\mathbf{v}}_{K}^{1}=\hat{\mathbf{v}}_{u E}^{1}=\left[\hat{u}_{X}^{1}, \hat{u}_{Y}^{1}, \hat{u}_{Z}^{1}\right]^{\mathrm{T}}=\mathbf{0}\left(\text { or } \hat{\mathbf{v}}_{K}^{1}=\hat{\mathbf{v}}_{u E}^{1}=\left[\hat{u}_{X}^{1}, \hat{u}_{Y}^{1}, \hat{u}_{Z}^{1}, \hat{\varphi}_{Z}^{1}\right]^{\mathrm{T}}=\mathbf{0}\right) \\
\mathbf{A}^{12}=\boldsymbol{\Phi}_{u-}^{12}, \mathbf{D}^{12}=\boldsymbol{\Phi}_{u+}^{12}
\end{aligned}
$$

For mixed boundary conditions, the form of known quantities and coefficient matrices can also be worked out accordingly. The boundary conditions on the bottom surface can be similarly deduced and will not be discussed for brevity.

Eq. (25) can be further written in a form of local scattering relations on the top and bottom surfaces

$$
\mathbf{A}^{1} \mathbf{a}^{1}+\mathbf{D}^{1} \mathbf{d}^{1}=\mathbf{s}_{0}^{1}, \mathbf{A}^{N+1} \mathbf{a}^{N+1}+\mathbf{D}^{N+1} \mathbf{d}^{N+1}=\mathbf{s}_{0}^{(N+1)}
$$

where $\mathbf{a}^{1}=\mathbf{a}^{12}\left(\mathbf{a}^{N+1}=\mathbf{a}^{(N+1) N}\right)$ and $\mathbf{d}^{1}=\mathbf{d}^{12}\left(\mathbf{d}^{N+1}=\mathbf{d}^{(N+1) N}\right)$ are the arriving and departing wave vectors of the top (bottom) surface, $\mathbf{A}^{1}=\mathbf{A}^{12} \quad\left(\mathbf{A}^{N+1}=\mathbf{A}^{(N+1) N}\right)$ and $\mathbf{D}^{1}=\mathbf{D}^{12}$ $\left(\mathbf{D}^{N+1}=\mathbf{D}^{(N+1) N}\right)$ are $n_{v} / 2 \times n_{a}^{12}\left(n_{v} / 2 \times n_{a}^{(N+1) N}\right)$ and $n_{v} / 2 \times n_{d}^{12}\left(n_{v} / 2 \times n_{d}^{(N+1) N}\right)$ coefficient matrices corresponding to the arriving and departing wave vectors of the top (bottom) surface, respectively.

It should be pointed out that the form of local scattering relations at the boundaries given in Eq. (30) is also valid for surface waves in a multilayered structure.

\subsubsection{Local scattering relations of a typical interface}
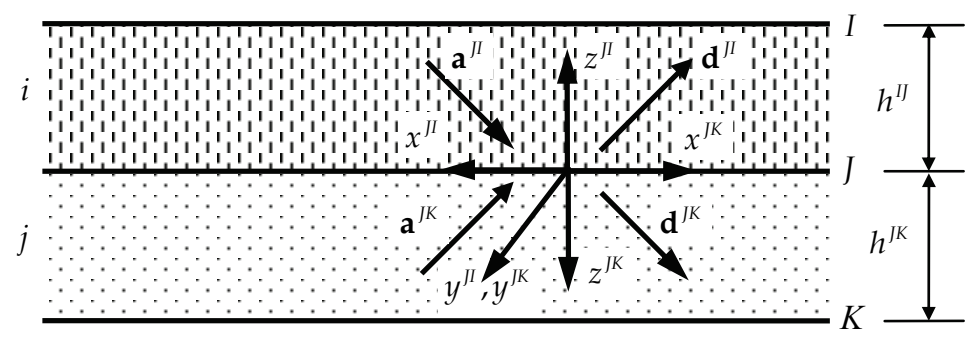

Fig. 3. A typical interface $J$ of the multilayered anisotropic structure 
Since the adjacent layers in the structure are perfectly bonded, the state variables should be continuous across the interfaces. Taking the typical interface $J$ as shown in Fig. 2 for illustration, the compatibility of the generalized displacements and equilibrium of the generalized forces require

$$
\mathbf{T}_{v} \hat{\mathbf{v}}^{I I}(0)=\hat{\mathbf{v}}^{J K}(0)
$$

This gives, according to the solutions in Eqs. (20) and (21),

$$
\left[\begin{array}{cc}
\mathbf{T}_{u} \boldsymbol{\Phi}_{u-}^{I I} & \mathbf{T}_{u} \boldsymbol{\Phi}_{u+}^{I I} \\
\mathbf{T}_{\sigma} \boldsymbol{\Phi}_{\sigma-}^{I I} & \mathbf{T}_{\sigma} \boldsymbol{\Phi}_{\sigma+}^{I I}
\end{array}\right]\left\{\begin{array}{l}
\mathbf{a}^{I I} \\
\mathbf{d}^{I I}
\end{array}\right\}=\left[\begin{array}{ll}
\boldsymbol{\Phi}_{u-}^{J K} & \boldsymbol{\Phi}_{u+}^{J K} \\
\boldsymbol{\Phi}_{\sigma-}^{J K} & \mathbf{\Phi}_{\sigma+}^{J K}
\end{array}\right]\left\{\begin{array}{c}
\mathbf{a}^{J K} \\
\mathbf{d}^{J K}
\end{array}\right\}
$$

It should be noticed once again that there is no exponential functions in the coupling equation (32) for interfaces. By grouping the arriving and departing wave vectors of relevant layers into the local arriving and departing wave vectors of the interface $\mathbf{a}^{J}=\left[\left(\mathbf{a}^{I I}\right)^{\mathrm{T}},\left(\mathbf{a}^{J K}\right)^{\mathrm{T}}\right]^{\mathrm{T}}$ and $\mathbf{d}^{J}=\left[\left(\mathbf{d}^{I I}\right)^{\mathrm{T}},\left(\mathbf{d}^{J K}\right)^{\mathrm{T}}\right]^{\mathrm{T}}$, Eq. (32) is reduced to the local scattering relation of the typical interface $J$

$$
\mathbf{A}^{J} \mathbf{a}^{J}+\mathbf{D}^{J} \mathbf{d}^{J}=\mathbf{0}
$$

where the $n_{v} \times\left(n_{a}^{I I}+n_{a}^{J K}\right)$ and $n_{v} \times\left(n_{d}^{I I}+n_{d}^{J K}\right)$ coefficient matrices $\mathbf{A}^{J}$ and $\mathbf{D}^{I}$, respectively, are

$$
\mathbf{A}^{J}=\left[\begin{array}{cc}
\mathbf{T}_{u} \boldsymbol{\Phi}_{u-}^{J I} & -\boldsymbol{\Phi}_{u-}^{J K} \\
\mathbf{T}_{\sigma} \boldsymbol{\Phi}_{\sigma-}^{J I} & -\boldsymbol{\Phi}_{\sigma-}^{J K}
\end{array}\right], \mathbf{D}^{J}=\left[\begin{array}{cc}
\mathbf{T}_{u} \boldsymbol{\Phi}_{u+}^{I I} & -\boldsymbol{\Phi}_{u+}^{J K} \\
\mathbf{T}_{\sigma} \boldsymbol{\Phi}_{\sigma+}^{J I} & -\boldsymbol{\Phi}_{\sigma+}^{J K}
\end{array}\right]
$$

There are altogether $N-1(\mathrm{n}-1)$ interfaces in the multilayered structure, so that we have $N-1(n-1)$ local scattering equations like Eq. (33).

\subsubsection{Global scattering relation of the structure}

The local scattering relations of top surface, interfaces and bottom surface have respectively $n_{v} / 2, n_{v} \times(N-1)$ and $n_{v} / 2$ equations, which are grouped together from up to down to give the $n_{v} \times N$ global scattering relation

$$
\mathrm{Aa}+\mathrm{Dd}=\mathbf{s}_{0}
$$

where the global arriving and departing wave vectors $\mathbf{a}$ and $\mathbf{d}$ are

$$
\begin{gathered}
\mathbf{a}=\left[\left(\mathbf{a}^{12}\right)^{\mathrm{T}},\left(\mathbf{a}^{21}\right)^{\mathrm{T}},\left(\mathbf{a}^{23}\right)^{\mathrm{T}}, \cdots,\left(\mathbf{a}^{J I}\right)^{\mathrm{T}},\left(\mathbf{a}^{J K}\right)^{\mathrm{T}}, \cdots,\left(\mathbf{a}^{N(N+1)}\right)^{\mathrm{T}},\left(\mathbf{a}^{(N+1) N}\right)^{\mathrm{T}}\right]^{\mathrm{T}} \\
\mathbf{d}=\left[\left(\mathbf{d}^{12}\right)^{\mathrm{T}},\left(\mathbf{d}^{21}\right)^{\mathrm{T}},\left(\mathbf{d}^{23}\right)^{\mathrm{T}}, \cdots,\left(\mathbf{d}^{I I}\right)^{\mathrm{T}},\left(\mathbf{d}^{J \mathrm{~K}}\right)^{\mathrm{T}}, \cdots,\left(\mathbf{d}^{N(N+1)}\right)^{\mathrm{T}},\left(\mathbf{d}^{(N+1) N}\right)^{\mathrm{T}}\right]^{\mathrm{T}}
\end{gathered}
$$

the corresponding $\left(n_{v} \times N\right) \times\left(n_{v} \times N\right)$ coefficient matrices $\mathbf{A}$ and $\mathbf{D}$ are

$$
\mathbf{A}=<\mathbf{A}^{1}, \mathbf{A}^{2}, \cdots, \mathbf{A}^{J}, \cdots, \mathbf{A}^{N+1}>, \mathbf{D}=<\mathbf{D}^{1}, \mathbf{D}^{2}, \cdots, \mathbf{D}^{J}, \cdots, \mathbf{D}^{N+1}>
$$

and $\mathbf{s}_{0}=\left[\left(\mathbf{s}_{0}^{1}\right)^{\mathrm{T}}, \mathbf{0}^{\mathrm{T}}, \cdots, \mathbf{0}^{\mathrm{T}},\left(\mathbf{s}_{0}^{(\mathrm{N}+1)}\right)^{\mathrm{T}}\right]^{\mathrm{T}}$ is the global excitation source vector. It should be noted that the forming process of scattering relations in Eqs. (30), (33) and (35) exclude matrix inversion as compared to that in the original formulation of MRRM (Pao et al, 2000, 2007; Su 
et al., 2002; Tian et al., 2006), which guarantees the numerical stability and at the same time enables the inclusion of surface and interface wave modes, in the proposed formulation of MRRM (Guo \& Chen, 2008a, 2008b; Guo, 2008; Guo et al., 2009).

\subsection{Phase relation from compatibility conditions of layers}

\subsubsection{Local phase relation of a typical layer}

Considering the formation of the dual local coordinates of a typical layer $J K(K J)$ as discussed in Section 3.1, we have the geometrical dual transformation relations

$$
x^{J K}=-x^{K J}, y^{J K}=y^{K J}, z^{K K}=h^{J K}-z^{K J}, \mathrm{~d} z^{J K}=-\mathrm{d} z^{K J}
$$

where $h^{J K}\left(=h^{K J}\right)$ represents the thickness of layer $J K(K J)$, and the physical dual transformation relations

$$
\hat{\mathbf{v}}^{J K}\left(z^{J K}\right)=\mathbf{T}_{v} \hat{\mathbf{v}}^{K J}\left(z^{K J}\right)
$$

By virtue of Eqs. (18) and (19), Eqs. (38) and (39), and the definitions of eigenvalue and eigenvector, it is derived that

$$
\mathbf{A}^{K J}\left(z^{K J}\right)=-\mathbf{T}_{v} \mathbf{A}^{J K}\left(z^{J K}\right) \mathbf{T}_{v}^{-1}, \Lambda^{J K}=-\boldsymbol{\Lambda}^{K J}, \boldsymbol{\Phi}^{J K}=\mathbf{T}_{v} \boldsymbol{\Phi}^{K J}
$$

It is interpreted that if $\lambda^{J K}$ and $\boldsymbol{\varphi}_{\lambda}^{J K}$ are the eigenvalue and eigenvector of the coefficient matrix $\mathbf{A}^{J K}$, then $-\lambda^{J K}$ and $\mathbf{T}_{v} \boldsymbol{\varphi}_{\lambda}^{J K}$ must be the corresponding eigenvalue and eigenvector of the coefficient matrix $\mathbf{A}^{K J}$. The equality relations between the numbers of arriving and departing waves in dual local coordinates, i.e. $n_{a}^{J K}=n_{d}^{K J}$ and $n_{d}^{J K}=n_{a}^{K J}$, are also implied. Substituting Eqs. (20) and (21) into Eq. (39), and in view of Eq. (40) and $\mathbf{T}_{v}=\mathbf{T}_{v}^{-1}$, one obtains the local phase relation of a typical layer $J K(K J)$

$$
\left\{\begin{array}{l}
\mathbf{a}^{J K} \\
\mathbf{a}^{K J}
\end{array}\right\}=\left[\begin{array}{cc}
\exp \left(-\boldsymbol{\Lambda}_{-}^{J K} h^{J K}\right) & \mathbf{0} \\
\mathbf{0} & \exp \left(\boldsymbol{\Lambda}_{+}^{J K} h^{J K}\right)
\end{array}\right]\left\{\begin{array}{l}
\mathbf{d}^{K J} \\
\mathbf{d}^{J K}
\end{array}\right\}=\left[\begin{array}{cc}
\mathbf{P}^{J K} & \mathbf{0} \\
\mathbf{0} & \mathbf{P}^{K J}
\end{array}\right]\left[\begin{array}{cc}
\mathbf{0} & \mathbf{I}_{a}^{J K} \\
\mathbf{I}_{d}^{I K} & \mathbf{0}
\end{array}\right]\left\{\begin{array}{l}
\mathbf{d}^{J K} \\
\mathbf{d}^{K J}
\end{array}\right\}
$$

where the $n_{a}^{J K} \times n_{a}^{J K}$ and $n_{d}^{J K} \times n_{d}^{J K}$ diagonal matrices $\mathbf{P}^{J K}=\exp \left(-\boldsymbol{\Lambda}_{-}^{J K} h^{J K}\right)$ and $\mathbf{P}^{K J}=\exp \left(\boldsymbol{\Lambda}_{+}^{J K} h^{J K}\right)$ are referred to as local phase matrices, and $\mathbf{I}_{a}^{J K}$ and $\mathbf{I}_{d}^{J K}$ are identity matrices of order $n_{a}^{J K}$ and $n_{d}^{J K}$, respectively. It should be noted that the exponentially growing functions, which usually cause numerical instability (such as in the TMM) for large values of the frequency-thickness product, have been completely excluded from the phase matrices $\mathbf{P}^{J K}$ and $\mathbf{P}^{K J}$, since we always have $\operatorname{Re}\left(\lambda_{-}^{J K} h^{J K}\right)>0$ or $\operatorname{Re}\left(\lambda_{-}^{J K} h^{J K}\right)=0, \operatorname{Im}\left(\lambda_{-}^{J K} h^{J K}\right)>0 \quad\left(\operatorname{Re}\left(\lambda_{+}^{J K} h^{J K}\right)<0 \quad\right.$ or $\left.\quad \operatorname{Re}\left(\lambda_{+}^{J K} h^{J K}\right)=0, \operatorname{Im}\left(\lambda_{+}^{J K} h^{J K}\right)<0\right)$. As indicated by Eq. (41), there are $n_{v}$ equations in the local phase relation of each layer.

\subsubsection{Global phase relation of the structure}

Grouping together the local phase relations for all layers from up to down yields the global phase relation with $n_{v} \times N$ equations

$$
\mathbf{a}=\mathbf{P} \overline{\mathbf{d}}=\mathbf{P U d}
$$

where the $\left(n_{v} \times N\right) \times\left(n_{v} \times N\right)$ block diagonal matrices $\mathbf{P}$, named the global phase matrix, is composed of 


$$
\mathbf{P}=<\mathbf{P}^{12}, \mathbf{P}^{21}, \mathbf{P}^{23}, \cdots, \mathbf{P}^{I I}, \mathbf{P}^{J K}, \cdots, \mathbf{P}^{N(N+1)}, \mathbf{P}^{(N+1) N}>
$$

the variant of the global departing wave vector $\overline{\mathbf{d}}$ is related to the wave vector $\mathbf{d}$ by the $\left(n_{v} \times N\right) \times\left(n_{v} \times N\right)$ block diagonal matrix $\mathbf{U}$, which is referred to as the global permutation matrix, to account for the different sequence of components arrangement between $\overline{\mathbf{d}}$ and $\mathbf{d}$. The specific forms of $\mathbf{U}$ and $\overline{\mathbf{d}}$ are as follows

$$
\begin{gathered}
\mathbf{U}=<\mathbf{U}^{12}, \mathbf{U}^{23}, \cdots, \mathbf{U}^{I K}, \cdots, \mathbf{U}^{N(N+1)}>, \mathbf{U}_{n_{v} \times n_{v}}^{\mathrm{JK}}=\left[\begin{array}{cc}
\mathbf{0} & \mathbf{I}_{a}^{I K} \\
\mathbf{I}_{d}^{I K} & \mathbf{0}
\end{array}\right] \\
\overline{\mathbf{d}}=\left[\left(\mathbf{d}^{21}\right)^{\mathrm{T}},\left(\mathbf{d}^{12}\right)^{\mathrm{T}},\left(\mathbf{d}^{32}\right)^{\mathrm{T}}, \cdots,\left(\mathbf{d}^{\mathrm{IJ}}\right)^{\mathrm{T}},\left(\mathbf{d}^{K J}\right)^{\mathrm{T}}, \cdots,\left(\mathbf{d}^{(N+1) N}\right)^{\mathrm{T}},\left(\mathbf{d}^{N(N+1)}\right)^{\mathrm{T}}\right]^{\mathrm{T}}
\end{gathered}
$$

It is seen from Eq. (42) that the global arriving and departing wave vectors a and $\mathbf{d}$, consisting of respectively the arriving and departing wave amplitudes in local dual coordinates of all layers and having the same forms as those in the global scattering relation in Eq. (36), are related directly through the global phase relation, which enables the dimension reduction of the system equation, making the final scale the same as the one in other analytical methods which are based on single local coordinates.

\subsection{System equation and dispersion equation}

The global scattering relation in Eq. (35) and global phase relation in Eq. (42) contain respectively $n_{v} \times N$ equations for the $n_{v} \times N$ unknown arriving wave amplitudes (in a ) and $n_{v} \times N$ unknown departing wave amplitudes (in $\mathbf{d}$ ). Thus the wave vectors can be determined. Substitution of Eq. (42) into Eq. (35) gives the system equation

$$
(\mathbf{A P U}+\mathbf{D}) \mathbf{d}=\mathbf{R d}=\mathbf{s}_{0}
$$

where $\mathbf{R}=\mathbf{A P U}+\mathbf{D}$ is the system matrix.

If there is no surface excitation, i.e. $\mathbf{s}_{0}=0$ and the free wave propagation problem is considered, the vanishing of the system matrix determinant yields the following dispersion equation

$$
\left|\mathbf{R}\left(k_{x} ; k_{y} ; \omega\right)\right|=\mathbf{0}
$$

which may be solved numerically by a proper root searching technique (Guo, 2008). Thus, the complete dispersion curves of various waves can be obtained, as illustrated in Section 4 for multilayered anisotropic elastic structures.

If there is surface excitation, from Eq. (46) we have

$$
\mathbf{d}=(\mathbf{A P U}+\mathbf{D})^{-1} \mathbf{s}_{0}=\mathbf{R}^{-1} \mathbf{s}_{0}
$$

Further making use of the global phase relation (42), the solution of the state vector in Eq. (20) and the inverse Fourier transform in Eq. (10) with respect to the wavenumbers, the steady-state response of state variables of a layer at circular frequency $\omega$ can be expressed as

$$
\begin{aligned}
& \hat{\mathbf{v}}^{I K}\left(x^{J K}, y^{J K}, z^{I K} ; \omega\right)=\frac{1}{(2 \pi)^{2}} \int_{-\infty}^{+\infty} \int_{-\infty}^{+\infty} \hat{\mathbf{v}}^{I K}\left(k_{x} ; k_{y}, z^{J K} ; \omega\right) \mathrm{e}^{\mathrm{i}\left(k_{x} x^{I K}+k_{y} y^{I K}\right)} \mathrm{d} k_{x} \mathrm{~d} k_{y} \\
& \left.=\frac{1}{(2 \pi)^{2}} \int_{-\infty}^{+\infty} \int_{-\infty}^{+\infty}\left\{\boldsymbol{\Phi}_{-}^{J K} \exp \left(\boldsymbol{\Lambda}_{-}^{J K} z^{J K}\right) \mathbf{E}^{K J}+\boldsymbol{\Phi}_{+}^{J K} \exp \left(\boldsymbol{\Lambda}_{+}^{J K} z^{J K}\right) \mathbf{E}^{J K}\right\} \mathbf{R}^{-1} \mathbf{s}_{0} \mathrm{e}^{\mathrm{i}\left(k_{x} x^{I K}+k_{y} y^{J K}\right.}\right) \mathrm{d} k_{x} \mathrm{~d} k_{y}
\end{aligned}
$$


and the transient response should be obtained from the corresponding steady-state response by means of inverse Fourier transform with respect to frequency as

$$
\begin{aligned}
& \mathbf{v}^{J K}\left(x^{J K}, y^{J K}, z^{J K}, t\right)=\frac{1}{2 \pi} \int_{-\infty}^{+\infty} \hat{\mathbf{v}}^{J K}\left(x^{J K}, y^{J K}, z^{J K} ; \omega\right) \mathrm{e}^{\mathrm{i} \omega t} \mathrm{~d} \omega \\
& =\frac{1}{(2 \pi)^{3}} \int_{-\infty}^{+\infty} \int_{-\infty}^{+\infty} \int_{-\infty}^{+\infty}\left\{\boldsymbol{\Phi}_{-}^{J K} \exp \left(\boldsymbol{\Lambda}_{-}^{J K} z^{J K}\right) \mathbf{E}^{K J}+\boldsymbol{\Phi}_{+}^{J K} \exp \left(\boldsymbol{\Lambda}_{+}^{J K} z^{J K}\right) \mathbf{E}^{J K}\right\} \mathbf{R}^{-1} \mathbf{s}_{0} \mathrm{e}^{\mathrm{i}\left(k_{x} x^{J K}+k_{y} y^{J K}+\omega t\right)} \mathrm{d} k_{x} \mathrm{~d} k_{y} \mathrm{~d} \omega
\end{aligned}
$$

Eqs. (49) and (50) can be solved numerically by the FFT technique (Guo, 2008).

\section{Numerical examples}

To verify the proposed method, we consider laminated composites with reinforced fibers alternately aligning along the $x$-axis $\left(0^{\circ}\right)$ and the $y$-axis $\left(90^{\circ}\right)$. The material properties of a lamina with fibers in $0^{\circ}$ and $90^{\circ}$ directions are given in Table 1.

\begin{tabular}{ccccccccccc}
\hline Lamina & $\rho$ & $c_{11}$ & $c_{12}$ & $c_{13}$ & $c_{22}$ & $c_{23}$ & $c_{33}$ & $c_{44}$ & $c_{55}$ & $c_{66}$ \\
\hline $0^{\circ}$ & 1200 & 1.6073 & 0.0644 & 0.0644 & 0.1392 & 0.0692 & 0.1392 & 0.035 & 0.0707 & 0.0707 \\
$90^{\circ}$ & 1200 & 0.1392 & 0.0644 & 0.0692 & 1.6073 & 0.0644 & 0.1392 & 0.0707 & 0.035 & 0.0707 \\
\hline
\end{tabular}

Note: The unit of density is $\mathrm{kg} / \mathrm{m}^{3}$ and that of stiffness is $10^{11} \mathrm{~N} / \mathrm{m}^{2}$.

Table 1. Material properties of $0^{\circ}$ and $90^{\circ}$ laminas

In the previous work (Guo \& Chen, 2008a), dispersion curves have been calculated for a single layer of $0^{\circ}$ lamina and a triple-layered structure with a $0^{\circ} / 90^{\circ} / 0^{\circ}$ configuration with equal thickness of each lamina. The obtained results were compared with those obtained by the finite element stiffness method (Datta et al., 1988) (Figs. 4 and 8 for single layer and Figs. 5 and 9 for triple layers therein). The excellent agreement validates our derivation and the computer codes. Nevertheless, it should be pointed out that the proposed MRRM is analytical, based on continuous (distributed-parameter) model. Thus, it can give more accurate results but at less computational expense especially in the high-frequency range. Recently, the characteristics of free waves in single PZT-4 and/or barium sodium niobate (BSN) layers have been discussed (Guo et al., 2009) for different boundary conditions, and dispersion curves of bi-layered, triple-layered and ten-layered piezoelectric structures composed of alternate PZT-4 and BSN layers with equal thickness were also presented (Guo \& Chen, 2008b; Guo et al., 2009).

Here in this chapter, a four-layered composite with a $0^{\circ} / 90^{\circ} / 0^{\circ} / 90^{\circ}$ configuration is considered. The waves are assumed to propagate in the $\mathrm{X}$-direction for illustration, i.e. $k_{x}=k$ and $k_{y}=0$. Note that the formulations established in previous sections are valid for waves propagating in any direction in the XOY plane. For the sake of presentation, we define the dimensionless quantities, including frequency $\Omega$, wavenumber $\gamma$, wavelength $L$ and phase velocity $V$ by $\Omega=\omega H /\left(2 \pi c_{s}\right), \quad \gamma=k H /(2 \pi), L=2 \pi \lambda / H=4 \pi^{2} /(k H)$ and $V=c / c_{s}$, respectively, where $H$ is the total thickness of the composite plate, $k, \lambda$ and $c$ are respectively the wavenumber, wavelength and phase velocity, $c_{s}=\sqrt{\left(c_{55} / \rho\right)_{0^{\circ}}}$ is the shear wave velocity constant with $\left(c_{55}\right)_{0^{\circ}}$ and $(\rho)_{0^{\circ}}$ the stiffness coefficient and material density of $0^{\circ}$ lamina. The thickness of the $0^{\circ}$ and $90^{\circ}$ laminas are denoted as $h_{1}$ and $h_{2}$, respectively. 


\subsection{Dispersion curves of multilayered anisotropic structures with free surfaces}

First, the laminas of the four-layered composite structure are assumed to have equal thicknesses and the top and bottom surfaces of the composite are assumed to be tractionfree. The dispersion curves, in terms of frequency-wavenumber spectra, wavelengthfrequency spectra and phase velocity-frequency spectra, are presented in Figs. 4(a), 4(b) and $4(c)$ respectively.

The sub-figures (a) to (c) in Fig. 4 show similar dispersion properties of free waves in the four-layered composite as compared with those for single and triple layers. The quasi P-SV and $\mathrm{SH}$ bulk modes, surface and interface modes and characteristic asymptotic line are obtained all at once from the dispersion equation by a root searching algorithm.

\subsection{Dispersion curves in high frequency range}

The frequency-wavenumber, wavelength-frequency and phase velocity-frequency spectra with dimensionless frequency $\Omega$ in the range of 100 to 102 are given in Figs. 5(a), 5(b) and 5(c) respectively, which indicate the proposed formulation of MRRM can assure a good numerical stability in the high-frequency range. Since the wave modes at small values of wavelength and phase velocity are relatively intensive and difficult to differentiate within this frequency range, as implied in Figs. 4(b) and 4(c), the dimensionless wavelength $L$ and phase velocity $V$ are specified within $0.75 \sim 2.00$ and 10 50 in Figs. 5(b) and 5(c), respectively.

\subsection{Effects of configuration on the dispersion curves}

Next, the thickness of the $0^{\circ}$ and $90^{\circ}$ laminas of the four-layered composite are assumed to be unequal in order to study the effect of configuration on the characteristics of free waves. The dispersion curves for cases $h_{1} / h_{2}=1 / 4$ and $h_{1} / h_{2}=4 / 1$ as well as their comparison with those for the equal thickness case (denoted as $h_{1} / h_{2}=1 / 1$ ) are depicted in Fig. 6, with the frequency-wavenumber, wavelength-frequency and phase velocity-frequency spectra given in the sub-figures (a), (b) and (c) respectively.

It is seen from Fig. 6 that the dispersion curves of a specified wave mode corresponding to the case $h_{1} / h_{2}=1 / 1$ locate in between those for cases $h_{1} / h_{2}=1 / 4$ and $h_{1} / h_{2}=4 / 1$. As also indicated in Fig. 6, the thickness ratio has a distinct effect on the characteristics of all free wave modes. The effect is however somehow larger for the higher-order wave modes than the lower-order ones.

\subsection{Effects of boundary conditions on the dispersion curves}

In order to show the effects of boundary conditions on the dispersion characteristics, the same $0^{\circ} / 90^{\circ} / 0^{\circ} / 90^{\circ}$ laminated composite with equal layer thicknesses is considered for two different boundary conditions: one is that both the top and bottom surfaces are fixed and the other is that the top surface is traction-free while the bottom surface is fixed. The dispersion curves for the two cases are given in Fig. 7 and compared with those for a laminate with free surfaces.

It is seen from Fig. 7 that some parts of the dispersion curves of certain specified modes for the four-layered composite with different surface conditions may coincide, but they may be completely different at other parts or for other modes. Fig. 7 indicates that the boundary conditions have a complex effect on the dispersion characteristics of free waves in multilayered anisotropic structures. In-depth study is needed. 


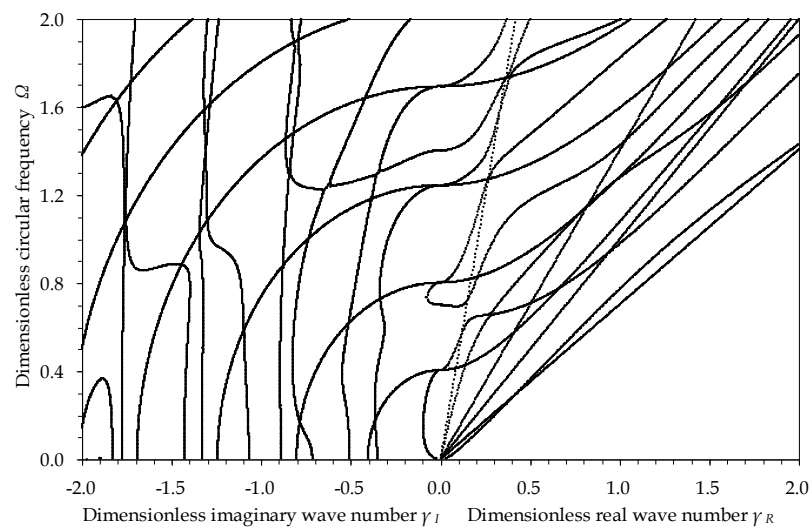

(a) Frequency-wavenumber spectra

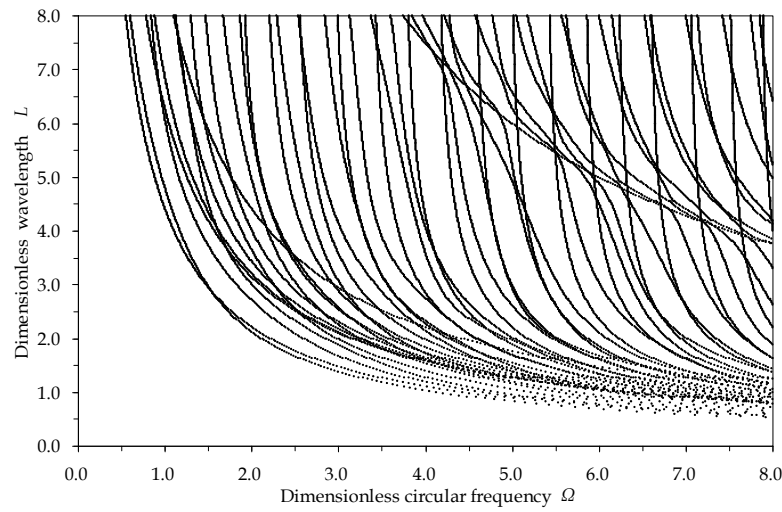

(b) Wavelength-frequency spectra

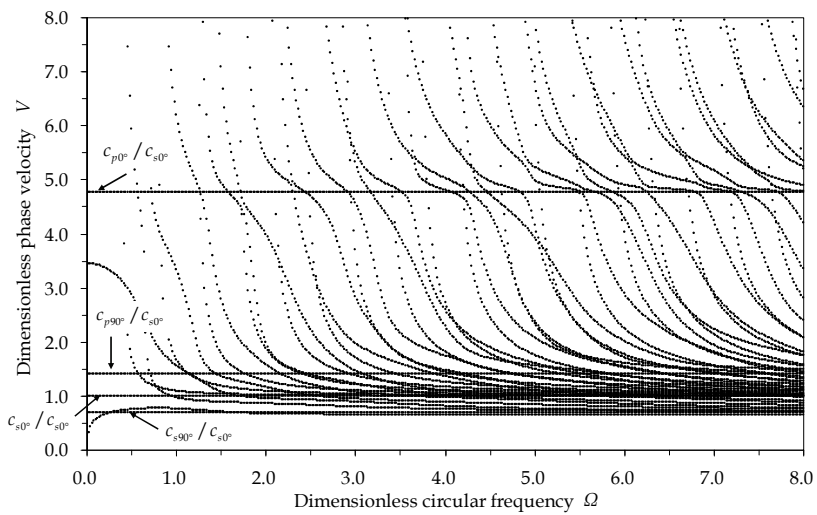

(c) Phase velocity-frequency spectra

Fig. 4. Dispersion curves of the $0^{\circ} / 90^{\circ} / 0^{\circ} / 90^{\circ}$ laminated composite with free surfaces 


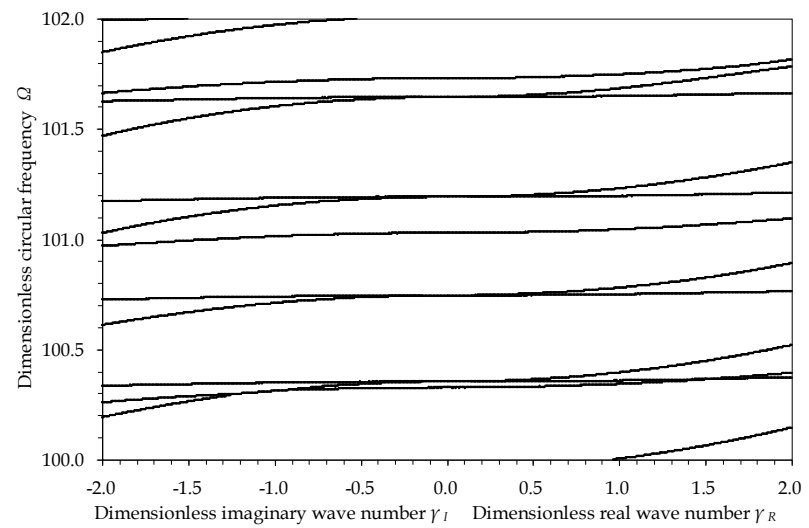

(a) Frequency-wavenumber spectra

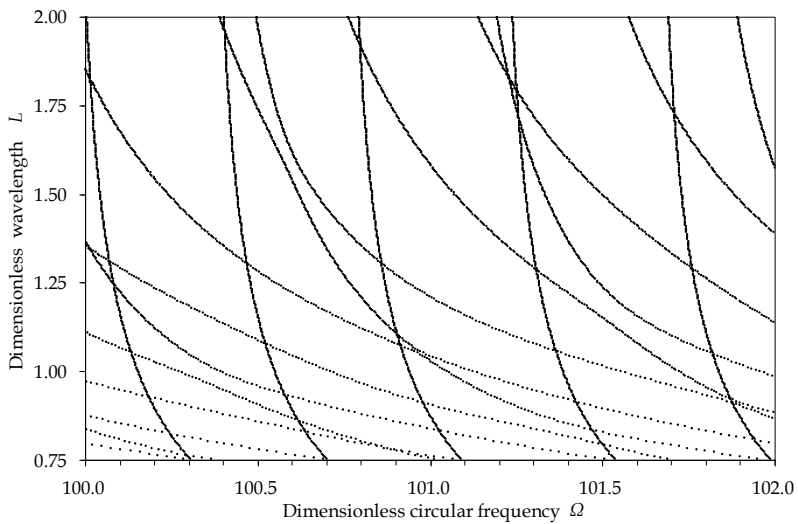

(b) Wavelength-frequency spectra

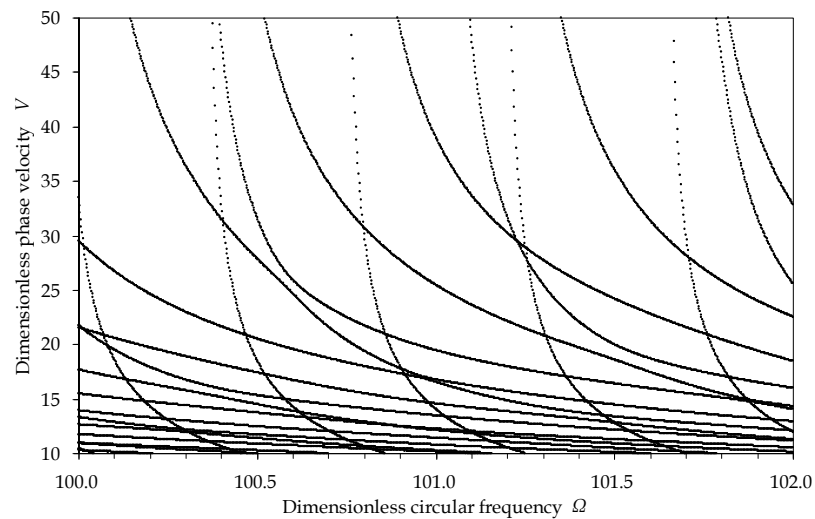

(c) Phase velocity-frequency spectra

Fig. 5. Dispersion curves at high frequency of the four-layered composite with free surfaces 


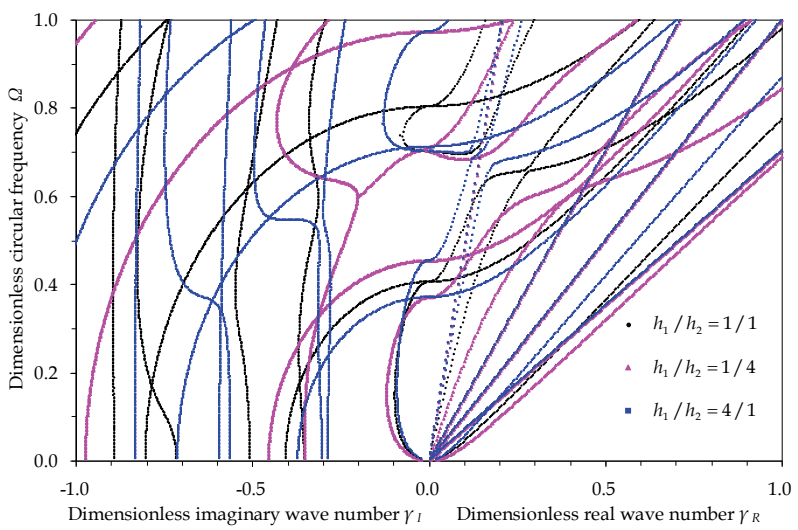

(a) Frequency-wavenumber spectra

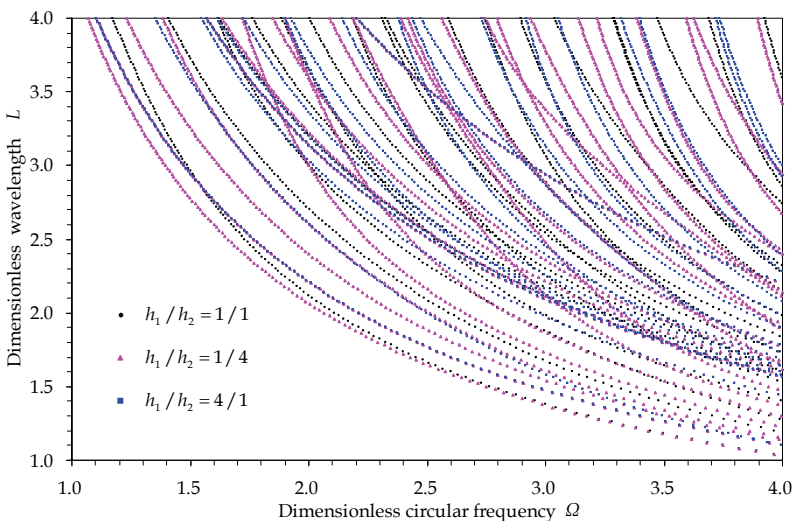

(b) Wavelength-frequency spectra

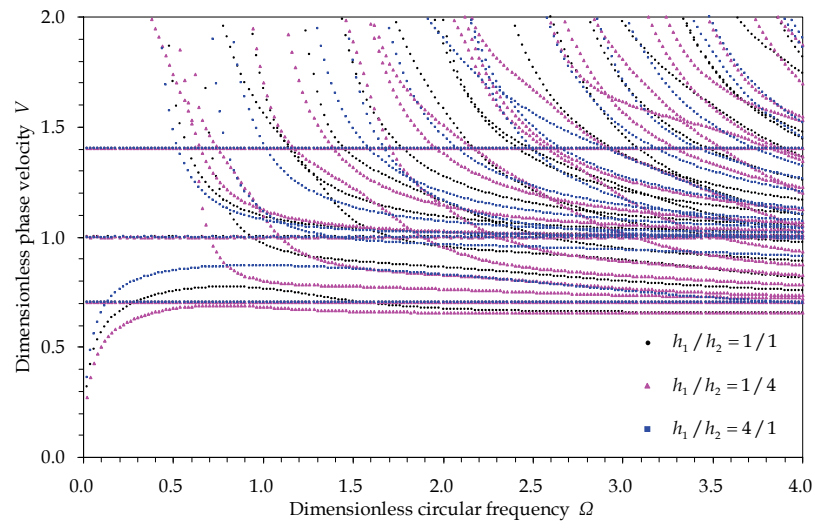

(c) Phase velocity-frequency spectra

Fig. 6. Comparisons of dispersion curves of the composite with different configurations 


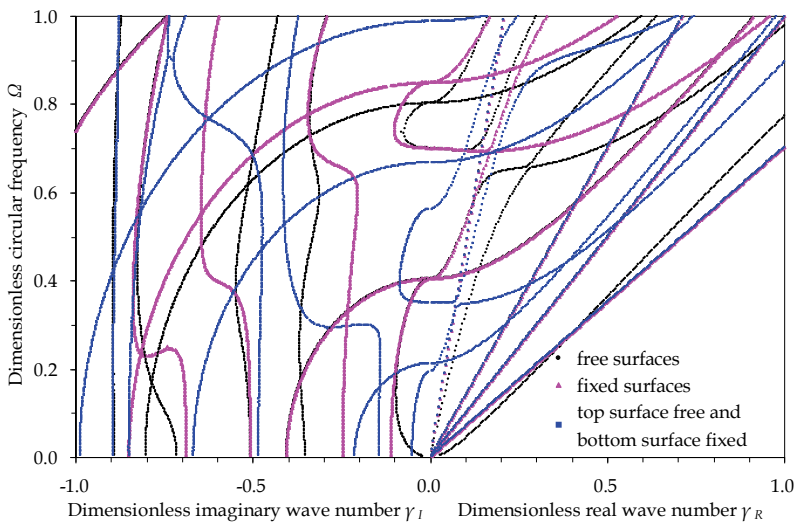

(a) Frequency-wavenumber spectra

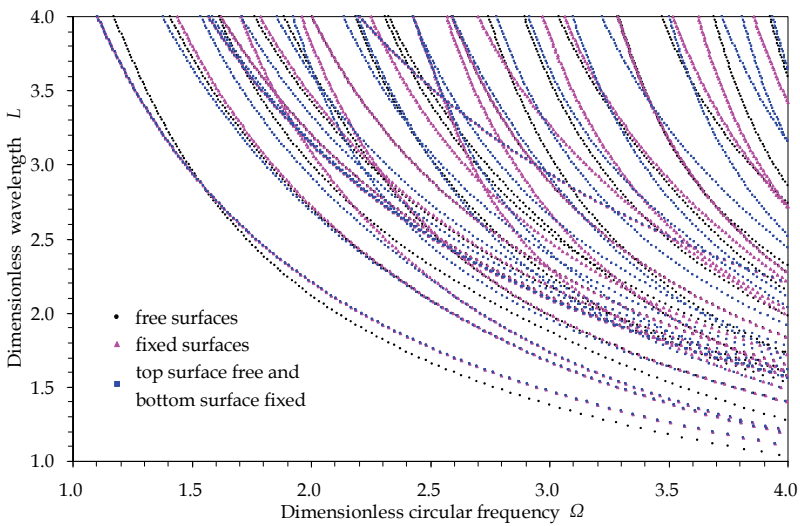

(b) Wavelength-frequency spectra

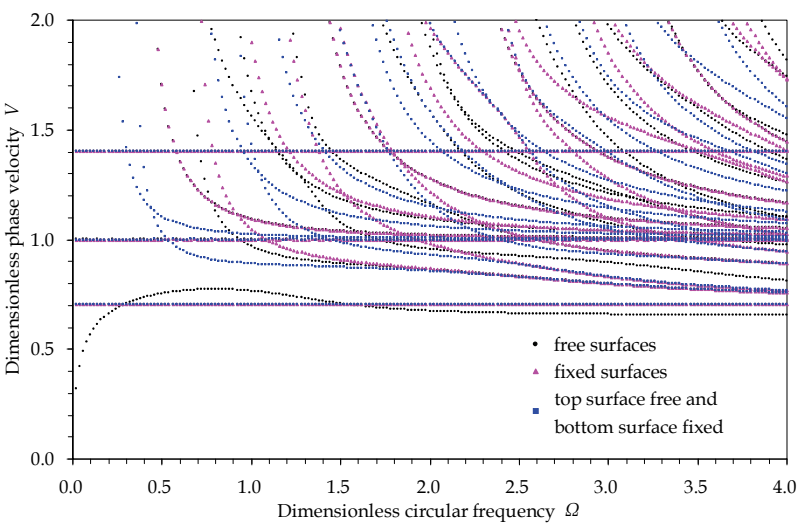

(c) Phase velocity-frequency spectra

Fig. 7. Comparison of dispersion curves of the composite with different boundary conditions 


\section{Conclusion}

We present a unified formulation of the method of reverberation-ray matrix (MRRM) for the analysis of acoustic wave propagation in multilayered anisotropic elastic/piezoelectric structures based on the state space formalism and Fourier transforms in the framework of three-dimensional elasticity or piezoelectricity. The proposed formulation of MRRM includes all wave modes in the structure and possesses good numerical stability by properly excluding exponentially growing function and matrix inversion operation. It is therefore suitable for the accurate analysis of acoustic waves in complex multilayered anisotropic structures by a uniform computer program. In comparison with the well-known traditional transfer matrix method, the present MRRM is unconditionally numerically stable, irrespective of the total number of layers, the thickness of individual layers and the frequency. Besides, in comparison with the numerical methods based on discrete models, the present MRRM is based on a continuous model (distributed-parameter model) and gives accurate results at a much smaller computational cost especially in the high-frequency range. Numerical results indicate a high accuracy and broad versatility of the proposed formulation of MRRM for wave propagation in multilayered anisotropic structures with various configurations and boundary conditions in any frequency range. The obtained dispersion curves and their dependence on the structural configurations and boundary conditions shall be useful in the design and optimization of laminated composites and acoustic wave devices.

\section{Acknowledgements}

This study was financially supported by the National Natural Science Foundation of China (No. 10902045 and No. 10725210) and the Postdoctoral Science Foundation of China (No. 20090460155) and the Fundamental Research Funds for the Central Universities of China.

\section{References}

Achenbach, J. D. (1973). Wave Propagation in Elastic Solids, North-Holland, Amsterdam.

Coddington, E. A., Levinson, N. (1955). Theory of Ordinary Differential Equations, McGrawHill, New York.

Adler, E. L. (1990). Matrix methods applied to acoustic waves in multilayers, IEEE Transactions on Ultrasonics, Ferroelectrics, and Frequency Control, 37(6): 485-490.

Adler, E. L. (2000). Bulk and surface acoustic waves in anisotropic solids, International Journal of High Speed Electronics and Systems, 10(3): 653-684.

Alshits V. I. \& Maugin, G. A. (2008). Dynamics of anisotropic multilayers, Wave Motion, 45(5): 629-640.

Auld, B. A. (1990). Acoustic Fields and Waves in Solids, Second Edition, Vol. I \& II, Robert E. Krieger Publishing Company, Malabar, Florida.

Brekhovskikh, L. M. (1980). Waves in Layered Media, Second Edition. Academic Press, New York.

Chakraborty, A. \& Gopalakrishnan, S. (2006). A spectral finite element model for wave propagation analysis in laminated composite plate, ASME Journal of Vibration and Acoustics, 128: 477-488.

Chimenti, D. E. (1997). Guided waves in plates and their use in materials characterization, Applied Mechanics Reviews, 50(5): 247-284.

Collet, B. (2004). Recursive surface impedance matrix methods for ultrasonic wave propagation in piezoelectric multilayers, Ultrasonics, 42: 189-197.

Datta, S. K., Shah, A. H., Bratton, R. L. \& Chakraborty, T. (1988). Wave propagation in laminated composite plates, Journal of the Acoustical Society of America, 83(6): 2020-2026. 
Degettekin, F. L., Honein, B. V., \& Khuri-Yakub, B. T. (1996). Application of surface impedance approach to ultrasonic wave propagation in layered anisotropic media, IEEE Ultrasonics Symposium, 559-562.

Ding, H. J. \& Chen, W. Q. (2001). Three Dimensional Problems of Piezoelasticity. Nova Science Publishers, New York.

Ewing, W. M., Jardetzky, W. S. \& Press, F. (1957). Elastic Waves in Layered Media. McGrawHill, New York.

Fedosov, V. I., Aniiimkin, V. I., Kotelyanskii, I. M., Caliendo, C., Verardi, P. \& Verona, E. (1996). Analysis of acoustic waves in multilayers using compound matrices, IEEE Ultrasonics Symposium, 207-212.

Guo, Y. Q. (2008). The Method of Reverberation-Ray Matrix and its Applications, Doctorial dissertation. Zhejiang University, Hangzhou, China. (in Chinese)

Guo, Y. Q. \& Chen, W. Q. (2008a). On free wave propagation in anisotropic layered media, Acta Mechanica Solida Sinica, 21(6): 500-506.

Guo, Y. Q. \& Chen, W. Q. (2008b) Modeling of multilayered acoustic wave devices with the method of reverberation-ray matrix, 2008 Symposium on Piezoelectricity, Acoustic Waves, and Device Applications (SPAWDA 2008), 105-110.

Guo, Y. Q., Chen, W. Q. \& Zhang, Y. L. (2009). Guided wave propagation in multilayered piezoelectric structures, Science in China, Series G: Physics, Mechanics and Astronomy, 52(7): 1094-1104.

Haskell, N. A. (1953). The dispersion of surface waves on multilayered media, Bulletin of the Seismological Society of America, 43: 17-34.

Honein, B., Braga, A. M. B., Barbone, P. \& Herrmann, G. (1991). Wave propagation in piezoelectric layered media with some applications, Journal of Intelligent Material Systems and Structures, 2: 542-557.

Hosten, B. \& Castaings, M. (2003). Surface impedance matrices to model the propagation in multilayered media, Ultrasonics, 41: 501-507.

Igel, H., Mora, P. \& Riollet, B. (1995). Anisotropic wave propagation through finitedifference grids, Geophysics, 60(4): 1203-1216.

Ingebrigtsen, K. A. \& Tonning, A. (1969). Elastic surface waves in crystal, Physical Review, 184: 942-951.

Kausel, E. \& Roesset, J. M. (1981). Stiffness matrices for layered soils, Bulletin of the Seismological Society of America, 71: 1743-1761.

Kennett, B. L. N. (1983). Seismic Wave Propagation in Stratified Media, Cambridge University Press, Cambridge.

Lowe, M. J. S. (1995). Matrix techniques for modeling ultrasonic waves in multilayered media, IEEE Transactions on Ultrasonics, Ferroelectrics, and Frequency Control, 42: 525-542.

Makkonen, T. (2005). Numerical Simulations of Microacoustic Resonators and Filters, Doctoral Dissertation. Helsinki University of Technology, Espoo, Finland.

Nayfeh, A. H. (1995). Wave Propagation in Layered Anisotropic Media with Applications to Composites. Elsevier, Amsterdam.

Pao, Y. H., Chen, W. Q. \& Su, X. Y. (2007). The reverberation-ray matrix and transfer matrix analyses of unidirectional wave motion, Wave Motion, 44: 419-438.

Pao, Y. H., Su, X. Y. \& Tian, J. Y. (2000). Reverberation matrix method for propagation of sound in a multilayered liquid, Journal of Sound and Vibration, 230(4): 743-760.

Pastureaud, T., Laude, V. \& Ballandras S. (2002). Stable scattering-matrix method for surface acoustic waves in piezoelectric multilayers, Applied Physics Letters, 80: 2544-2546.

Rizzi, S. A. \& Doyle, J. F. (1992). A spectral element approach to wave motion in layered solids, ASME Journal of Vibration and Acoustics, 114: 569-577. 
Rokhlin, S. I. \& Wang, L. (2002a). Stable recursive algorithm for elastic wave propagation in layered anisotropic media: Stiffness matrix method, Journal of the Acoustical Society of America, 112: 822-834.

Rokhlin, S. I. \& Wang, L. (2002b). Ultrasonic waves in layered anisotropic media: characterization of multidirectional composites, International Journal of Solids and Structures, 39(16): 4133-4149.

Rose, J. L. (1999). Ultrasonic Waves in Solid Media, Cambridge University Press, Cambridge.

Shen, S. P., Kuang, Z. B. \& Hu, S.L. (1998). Wave propagation in multilayered anisotropic media, Mechanics Research Communications, 25(5): 503-507.

Stroh, A. N. (1962). Steady state problems in anisotropic elasticity, Journal of Mathematics and Physics, 41: 77-103.

$\mathrm{Su}, \mathrm{X}$. Y., Tian, J. Y. \& Pao, Y. H. (2002). Application of the reverberation-ray matrix to the propagation of elastic waves in a layered solid, International Journal of Solids and Structures, 39: 5447-5463.

Synge, J. L. (1956). Flux of energy for elastic waves in anisotropic media, Proceedings of the Royal Irish Academy, 58: 13-21.

Tan, E. L. (2005). Stiffness matrix method with improved efficiency for elastic wave propagation in layered anisotropic media, Journal of the Acoustical Society of America, 118(6): 3400-3403.

Tan, E. L. (2006). Hybrid compliance-stiffness matrix method for stable analysis of elastic wave propagation in multilayered anisotropic media, Journal of the Acoustical Society of America, 119(1): 45-53.

Tan, E. L. (2007). Matrix Algorithms for modeling acoustic waves in piezoelectric multilayers, IEEE Transactions on Ultrasonics, Ferroelectrics, and Frequency Control, 54: 2016-2023.

Tarn, J. Q. (2002a). A state space formalism for anisotropic elasticity. Part I: Rectilinear anisotropy, International Journal of Solids and Structures, 39: 5143-5155

Tarn, J. Q. (2002b). A state space formalism for piezothermoelasticity, International Journal of Solids and Structures, 39: 5173-5184

Thomson, T. (1950). Transmission of elastic waves through a stratified solid medium, Journal of Applied Physics, 21: 89-93.

Tian, J. Y., Yang, W. X. \& Su, X. Y. (2006). Transient elastic waves in a transversely isotropic laminate impacted by axisymmetric load, Journal of Sound and Vibration, 289: 94-108.

Wang, L. \& Rokhlin, S. I. (2001). Stable reformulation of transfer matrix method for wave propagation in layered anisotropic media, Ultrasonics, 39: 413-424.

Wang, L. \& Rokhlin, S. I. (2002a). An efficient stable recursive algorithm for elastic wave propagation in layered anisotropic media, in Thompson, D. O. \& Chimenti, D. E. (ed.), Review of Quantitative Nondestructive Evaluation 21, pp. 115-122.

Wang, L. \& Rokhlin, S. I. (2002b). Recursive asymptotic stiffness matrix method for analysis of surface acoustic wave devices on layered piezoelectric media, Applied Physics Letters, 81: 4049-4051.

Wang, L. \& Rokhlin, S. I. (2004a). A compliance/stiffness matrix formulation of general Green's function and effective permittivity for piezoelectric multilayers, IEEE Transactions on Ultrasonics, Ferroelectrics, and Frequency Control, 51: 453-463.

Wang, L. \& Rokhlin, S. I. (2004b). A simple method to compute ultrasonic wave propagation in layered anisotropic media, in Thompson, D. O. \& Chimenti, D. E. (ed.), Review of Quantitative Nondestructive Evaluation 23, pp. 59-66.

Wang, L. \& Rokhlin, S. I. (2004c). Modeling of wave propagation in layered piezoelectric media by a recursive asymptotic method, IEEE Transactions on Ultrasonics, Ferroelectrics, and Frequency Control, 51: 1060-1071.

Zhang, V. Y., Lefebvre, J. E., Bruneel, C. \& Gryba, T. (2001). A unified formalism using effective surface permittivity to study acoustic waves in various anisotropic and piezoelectric multilayers, IEEE Transactions on Ultrasonics, Ferroelectrics, and Frequency Control, 48: 1449-1461. 


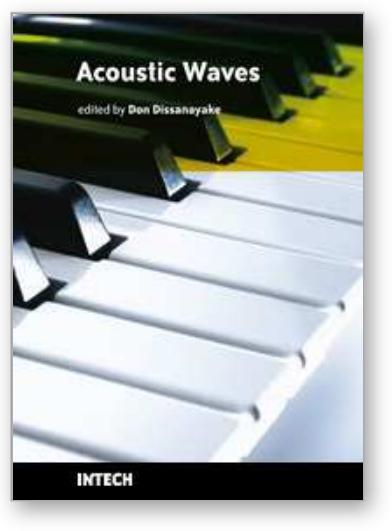

\author{
Acoustic Waves \\ Edited by Don Dissanayake
}

ISBN 978-953-307-111-4

Hard cover, 434 pages

Publisher Sciyo

Published online 28, September, 2010

Published in print edition September, 2010

SAW devices are widely used in multitude of device concepts mainly in MEMS and communication electronics. As such, SAW based micro sensors, actuators and communication electronic devices are well known applications of SAW technology. For example, SAW based passive micro sensors are capable of measuring physical properties such as temperature, pressure, variation in chemical properties, and SAW based communication devices perform a range of signal processing functions, such as delay lines, filters, resonators, pulse compressors, and convolvers. In recent decades, SAW based low-powered actuators and microfluidic devices have significantly added a new dimension to SAW technology. This book consists of 20 exciting chapters composed by researchers and engineers active in the field of SAW technology, biomedical and other related engineering disciplines. The topics range from basic SAW theory, materials and phenomena to advanced applications such as sensors actuators, and communication systems. As such, in addition to theoretical analysis and numerical modelling such as Finite Element Modelling (FEM) and Finite Difference Methods (FDM) of SAW devices, SAW based actuators and micro motors, and SAW based micro sensors are some of the exciting applications presented in this book. This collection of up-to-date information and research outcomes on SAW technology will be of great interest, not only to all those working in SAW based technology, but also to many more who stand to benefit from an insight into the rich opportunities that this technology has to offer, especially to develop advanced, low-powered biomedical implants and passive communication devices.

\title{
How to reference
}

In order to correctly reference this scholarly work, feel free to copy and paste the following:

Yongqiang Guo and Weiqiu Chen (2010). Reverberation-Ray Matrix Analysis of Acoustic Waves in Multilayered Anisotropic Structures, Acoustic Waves, Don Dissanayake (Ed.), ISBN: 978-953-307-111-4, InTech, Available from: http://www.intechopen.com/books/acoustic-waves/reverberation-ray-matrix-analysis-ofacoustic-waves-in-multilayered-anisotropic-structures

\section{INTECH}

open science | open minds

\section{InTech Europe}

University Campus STeP Ri

Slavka Krautzeka 83/A

51000 Rijeka, Croatia

\section{InTech China}

Unit 405, Office Block, Hotel Equatorial Shanghai

No.65, Yan An Road (West), Shanghai, 200040, China

中国上海市延安西路65号上海国际贵都大饭店办公楼 405 单元 
Phone: +385 (51) 770447

Fax: +385 (51) 686166

www.intechopen.com
Phone: +86-21-62489820

Fax: +86-21-62489821 
(C) 2010 The Author(s). Licensee IntechOpen. This chapter is distributed under the terms of the Creative Commons Attribution-NonCommercialShareAlike-3.0 License, which permits use, distribution and reproduction for non-commercial purposes, provided the original is properly cited and derivative works building on this content are distributed under the same license. 